\title{
Thermodynamic Properties of the Internal Rotation in Methyl Alcohol Vapor from 200 to $500^{\circ} \mathrm{K}$
}

\author{
J. O. HALFORD \\ Chemistry Department, University of Michigan, Ann Arbor, Michigan
}

(Received April 14, 1950)

\begin{abstract}
At ordinary and lower temperatures the thermodynamic properties of the methyl alcohol internal rotation are beyond the limits of available tabulations. For any internal rotator, of a given symmetry, with a fixed moment of inertia and potential barrier, two limiting values of each property can be calculated for any chosen temperature. Available tables are restricted to those cases for which the differences between the limiting values can be neglected. When, as with methyl alcohol, the differences are large, the representative values can be found by taking proper account of the symmetry and the dependence of the internal rotator energy levels upon the external rotations.

For methyl alcohol, the correct intermediate thermodynamic properties are obtained by using for the partition function and its derivatives the numerical mean of the limiting values of these sums obtained for the general rotator. This simple rule, however, is not necessarily applicable to other compounds.

Heat capacities and entropies, calculated from the spectroscopic potential barrier of $932 \mathrm{cal} . / \mathrm{mole}$ and other molecular constants, are considered in relation to experimental data.
\end{abstract}

$I_{\mathrm{i}}^{\mathrm{N}}$ a recent paper ${ }^{1}$ the height of the barrier to the internal rotation in methyl alcohol was recalculated from the difference between the third law entropy and the statistical entropy for free rotation. Substantially constant barrier calculations were obtained over a considerable temperature range, but deviations toward higher barriers were encountered both above and below the range of constancy. Such deviations are the expected result of inadequacy of the equation of state at the higher temperatures, but are probably due to other factors at lower temperatures. In particular, the low barrier and low moment of inertia cause this system to fall outside the limits of the entropy tables of Pitzer and Gwinn ${ }^{2}$ at low temperatures, with the result that the barrier must be obtained by an extrapolation which becomes more doubtful with decreasing temperature.

To remove this uncertainty from the barrier calculation would require an extension of the entropy tables, but, although as will be shown, this can be done for any one compound like methyl alcohol, the results will not necessarily be useful for other compounds. Instead, it is simpler to undertake a direct comparison of the third law entropy with the entropy from molecular constants, using, in the latter, the newly determined spectroscopic barrier of $326 \mathrm{~cm}^{-1}$ or $932 \mathrm{cal} . / \mathrm{mole}$. The evidence of consistency with changing temperature will then have been shifted from constancy of the calculated barrier to constancy of the difference between the molecular and third law entropies.

The energy levels of the internal rotator occur in bands which permit the calculation of two extreme values of each thermodynamic property between which the correct properties of any rotator must lie. Available tables, and, in fact, any accurate general tables, are limited to those regions of the variables for which the extreme calculated properties are not appreciably dif-

1 J. O. Halford, J. Chem. Phys, 18, 361 (1950).

2 K. S. Pitzer and W. D. Gwinn, J. Chem. Phys. 10, 428 (1942). ferent. Because the methyl alcohol system falls outside these limits there are appreciable differences, particularly in the heat capacity, between the extreme calculable properties, and a method must be found for establishing the valid intermediate values.

All of the necessary information about the energy levels is contained in the treatment of this system by Koehler and Dennison, ${ }^{3}$ who show how the particular set of internal levels varies with $K$, the quantum number for rotation of the rigid molecule around the axis of the internal rotation. With their theory it is possible to calculate all the rotational levels, internal and external, and consequently, to obtain the correct thermodynamic properties. The implied detailed calculation for all the rotations would be extremely tedious. It is possible, however, to make simplifications which eliminate much of the detail.

The partition function for rotation, both external and internal, can be written as

$$
Q=Q_{J} Q_{K} \tilde{q}
$$

where $Q_{J}$ refers to the external rotations with the large moments of inertia, $Q_{K}$ to the external rotation about an axis approximately parallel to the $\mathrm{CO}$ bond and $\tilde{q}$ is an effective average partition function for the internal rotation. $Q_{J}$ and $Q_{K}$ are equal to the classical partition functions at any temperature within the range of interest, and, further,

$$
Q_{K}=\sum_{K} \exp \left(-K^{2} \beta\right)
$$

with the summation taken over all integral values of $K$ between minus and plus infinity, in spite of the fact that the actual range of $K$ values is limited by $J$. It is therefore permissible to evaluate $Q_{J}$ and the product $Q_{K} \bar{q}$ independently, thus eliminating $Q_{J}$ from the present problem. There can be little doubt that for temperatures above $200^{\circ} \mathrm{K}$ the symmetry number approxima-

\footnotetext{
J. S. Koehler and D. M. Dennison, Phys. Rev. 57, 1006 (1940).
} 
TABLE I. Energy $\left(\Delta E / V_{0}\right)$ of the methyl alcohol internal rotation for $\alpha^{2}=6.0553$.

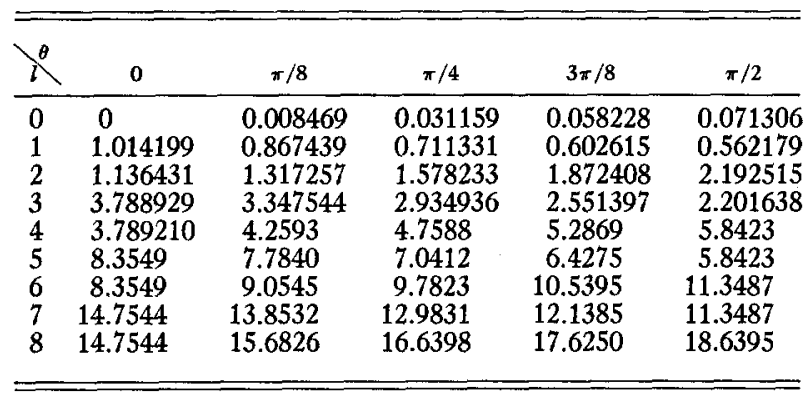

tion is accurate. This means that for each symmetry class of energy levels the valid average partition function for internal rotation must be the same or nearly the same, and may be expected to lie somewhere near the mean of the extreme calculable values. Exactly what mean value to take, however, is not evident in advance.

For the levels within a single symmetry class, let

$$
Q_{K} \bar{q}=\sum_{i K} \exp \left(-K^{2} \beta\right) \exp \left(-\epsilon_{i K}\right),
$$

with $\beta$ equal to $h^{2} / 8 \pi^{2} C k T$ where $C$ is the moment of inertia for external rotation, and $\epsilon_{i K}$ equal to $E_{i K} / k T$ where $E_{i K}$ is the excess of internal energy over that of the lowest possible internal rotation level. Since each $K$ defines a specific set of internal levels and, therefore, a partition function, $q_{K}$, it is permissible to sum over $i$ at constant $K$ to obtain

$$
\bar{q}=\left[\sum_{K} q_{K} \exp \left(-K^{2} \beta\right)\right] / Q_{K} .
$$

Similarly

$$
\begin{aligned}
Q^{\prime}=Q_{K}{ }^{\prime} \bar{q}+Q_{K} \bar{q}^{\prime} & \\
& =\sum_{i K}\left(K^{2} \beta+\epsilon_{i K}\right) \exp \left(-K^{2} \beta\right) \exp \left(-\epsilon_{i K}\right)
\end{aligned}
$$

which leads to

$$
\begin{aligned}
\bar{q}^{\prime}=\left[-Q_{K}^{\prime} \bar{q}+\sum_{K} q_{K} K^{2} \beta\right. & \exp \left(-K^{2} \beta\right) \\
& \left.+\sum_{K} q_{K}{ }^{\prime} \exp \left(-K^{2} \beta\right)\right] / Q_{K} .
\end{aligned}
$$

Finally,

$$
\begin{aligned}
Q^{\prime \prime}=Q_{K}{ }^{\prime \prime} \bar{q} & +Q_{K} \bar{q}^{\prime \prime}+2 Q_{K}{ }^{\prime} \bar{q}^{\prime} \\
& =\sum_{i K}\left(K^{2} \beta+\epsilon_{i K}\right)^{2} \exp \left(-K^{2} \beta\right) \exp \left(-\epsilon_{i K}\right)
\end{aligned}
$$

with the result that

$$
\begin{aligned}
\bar{q}^{\prime \prime}=\left[-Q_{K}^{\prime \prime} \bar{q}-2 Q_{K}^{\prime} \bar{q}^{\prime}\right. & +\sum_{K} q_{K} K^{4} \beta^{2} \exp \left(-K^{2} \beta\right) \\
& +2 \sum_{K} q_{K}{ }^{\prime} K^{2} \beta \exp \left(-K^{2} \beta\right) \\
& \left.+\sum_{K} q_{K}^{\prime \prime} \exp \left(-K^{2} \beta\right)\right] / Q_{K} .
\end{aligned}
$$

Equations (4), (6), and (8) give the average effective sums for the internal rotation from which the mean thermodynamic properties follow as $-F / T=R \ln \bar{q}, H / T$ $=R \bar{q}^{\prime} / \bar{q}, \quad S=R\left(\bar{q}^{\prime} / \bar{q}+\ln \bar{q}\right)$ and $C=R\left(\bar{q}^{\prime \prime} / \bar{q}-\left[\bar{q}^{\prime} / \bar{q}\right]^{2}\right)$. The required calculation is straightforward but very detailed. At room temperature the total number of individual states involved is of the order of 600 , divided equally among three symmetry classes. An approximation can be introduced, however, which is quite accurate down to very low temperatures and eliminates much of the detail.

The sums $q, q^{\prime}$, and $q^{\prime \prime}$ are required as functions of $K$. The energy levels are given by three periodic functions, one-third of them from each function. Each cycle is represented by three units on the scale $\sigma=-K C_{1} / C$ where $C_{1}$ is the moment of inertia of the $\mathrm{OH}$ group about the rotational axis. The three functions are the same except for a successive phase difference of one unit on the $\sigma$-scale. All the levels can be placed upon the same curve by the following device. Let $E_{i K}=P\left(\theta_{K}\right)$, where $\theta_{K}=\pi \sigma / 3$ for one-third of the levels, $\pi(\sigma+1) / 3$ for a second third, and $\pi(\sigma+2) / 3$ for the rest. The $\theta$-scale has been chosen, for convenience, so that the solutions repeat themselves at intervals of $\pi$-units, in order to avoid half-angles in the final expressions. The function

TABLe II. Partition function $q=\Sigma_{i} e^{-\epsilon_{i}}$ for $\alpha^{2}=6.0553$.

\begin{tabular}{cccccc}
\hline \hline$T$ & 0 & $\pi / 8$ & $\pi / 4$ & $3 \pi / 8$ & $\pi / 2$ \\
\hline 200 & 1.16259 & 1.15711 & 1.14389 & 1.13066 & 1.12518 \\
260 & 1.29146 & 1.28981 & 1.28582 & 1.28183 & 1.28018 \\
300 & 1.37948 & & & & 1.37453 \\
340 & 1.46624 & & & & 1.46410 \\
380 & 1.55075 & & & & 1.54983 \\
420 & 1.63267 & & & & 1.63229 \\
460 & 1.71198 & & & & 1.71182 \\
500 & 1.78874 & & & & \\
\hline
\end{tabular}

TABLE III. First derivative, $q^{\prime}=\Sigma_{i} \epsilon_{i} e^{-\epsilon_{i}}$ for $\alpha^{2}=6.0553$.

\begin{tabular}{cccccc}
\hline \hline$T^{\theta}$ & 0 & $\pi / 8$ & $\pi / 4$ & $3 \pi / 8$ & $\pi / 2$ \\
\hline 200 & 0.40843 & 0.42973 & 0.48119 & 0.53260 & 0.55390 \\
260 & 0.57235 & 0.58110 & 0.60225 & 0.62343 & 0.63224 \\
300 & 0.65722 & & & & 0.68812 \\
340 & 0.72873 & & & & 0.74407 \\
380 & 0.79079 & & & 0.79827 \\
420 & 0.84638 & & & 0.84992 \\
460 & 0.89712 & & & 0.89880 \\
500 & 0.94418 & & & 0.94498 \\
\hline
\end{tabular}

TABLE IV. Second derivative $q^{\prime \prime}=\Sigma_{i} \epsilon_{i}^{2} e^{-\epsilon_{i}}$ for $\alpha^{2}=6.0553$.

\begin{tabular}{cccccc}
\hline \hline$T^{\theta}$ & 0 & $\pi / 8$ & $\pi / 4$ & $3 \pi / 8$ & $\pi / 2$ \\
\hline 200 & 1.04052 & 1.00477 & 0.91864 & 0.83219 & 0.79642 \\
260 & 1.17902 & 1.15138 & 1.08473 & 1.01832 & 0.99123 \\
300 & 1.23759 & & & & 1.11051 \\
340 & 1.29211 & & & & 1.21397 \\
380 & 1.34710 & & & 1.30164 \\
420 & 1.40200 & & & & 1.37773 \\
460 & 1.45162 & & & & 1.44513 \\
500 & 1.51265 & & & & 1.50654 \\
\hline
\end{tabular}


can be set up by solving for enough energy levels to define the curve from $\theta=0$ to $\theta=\pi / 2$. Instead of outlining the function itself, it turns out to be simpler to set up $q_{K}, q_{K^{\prime}}$, and $q_{K}{ }^{\prime \prime}$ in terms of $\theta_{K}$.

In Table $I$, the internal energy levels are shown, for a sufficient number of the bands, at five points from $\theta=0$ to $\theta=\pi / 2$. They are expressed as $\Delta E / V_{0}$ where $\Delta E$ is the excess of energy above the lowest real level and $V_{0}$ is the height of the potential barrier measured from the bottom of the potential valley. The energies are derived from the potential energy function $V=V_{0}$ $(1-\cos 3 \varphi) / 2$ essentially by the methods described in an earlier paper, ${ }^{4}$ using $V_{0}=932 \mathrm{cal} . / \mathrm{mole}, C_{1}=1.01$ $\times 10^{-40}$, and, therefore, $\alpha=(9 / 4 \pi) Q_{f}{ }^{2} V / R T=(6.0553)^{\frac{1}{2}}$. In each column either four or five of the higher levels have been obtained by means of the old quantum theory approximation with the aid of a linear scale of fractional quantum numbers. For example, in the last row, at $l=8$, the successive quantum numbers are $4.0,4.125$, $4.250,4.375$, and 4.5 .

The energies of Table I have been used to obtain the sums $q=\sum_{i} e^{-\epsilon_{i}}$ (Table II), $q^{\prime}=\sum_{i \epsilon_{i}} e^{-{ }^{-i} i}$ (Table III) and $q^{\prime \prime}=\sum_{i} \epsilon_{i}{ }^{2} e^{-t i}$ (Table IV).

Examinations of the sums at 200 and $260^{\circ}$ in Tables II-IV reveals that, very closely,

$$
\begin{aligned}
q_{K} & =q_{0}+a \sin ^{2} \theta_{K}, \\
q_{K}^{\prime} & =q_{0}^{\prime}+b \sin ^{2} \theta_{K}, \\
q_{K}^{\prime \prime} & =q_{0}{ }^{\prime \prime}+c \sin ^{2} \theta_{K},
\end{aligned}
$$

where $q_{0}, q_{0}{ }^{\prime}$ and $q_{0}{ }^{\prime \prime}$ are obviously the sums for $\theta=0$, and $a, b$, and $c$ are constants. Equations (9) effect a considerable simplification of the required summations, and have led to the omission from the tables of the sums at higher temperatures and intermediate values of $\theta$, since these relations must become more accurate with increasing temperature and depend only upon the extreme values of the sums.

Equations (9) and the relations $Q_{K}{ }^{\prime} / Q_{K}=\frac{1}{2}$ and $Q_{K}{ }^{\prime \prime} / Q_{K}=\frac{3}{4}$ are substituted into Eqs. (2), (4), and (6) to yield the results,

$$
\begin{gathered}
\bar{q}=q_{0}+a\left[\sum_{K}\left(\sin ^{2} \theta_{K}\right) \exp \left(-K^{2} \beta\right)\right] / Q_{K} \\
\bar{q}^{\prime}=q_{0}{ }^{\prime}+\left(q_{0}-\bar{q}\right) / 2+\left[a \sum_{K}\left(\sin ^{2} \theta_{K}\right) K^{2} \beta \exp \left(-K^{2} \beta\right)\right. \\
\left.+b \sum_{K}\left(\sin ^{2} \theta_{K}\right) \exp \left(-K^{2} \beta\right)\right] / Q_{K} \\
\bar{q}^{\prime \prime}=q_{0}{ }^{\prime \prime}+3\left(q_{0}-\bar{q}\right) / 4+\left(q_{0}{ }^{\prime}-\bar{q}^{\prime}\right) \\
+\left[a \sum_{K}\left(\sin ^{2} \theta_{K}\right) K^{4} \beta^{2} \exp \left(-K^{2} \beta\right)\right. \\
+2 b \sum_{K}\left(\sin ^{2} \theta_{K}\right) K^{2} \beta \exp \left(-K^{2} \beta\right) \\
\left.+c \sum_{K}\left(\sin ^{2} \theta_{K}\right) \exp \left(-K^{2} \beta\right)\right] / Q_{K} .
\end{gathered}
$$

In Eqs. (10)-(12) the sums are taken over all values of $K$ from minus to plus infinity.

From Tables II-IV the constants of Eqs. (9)-(12) are found as shown in Table V.

The energy levels are now separated into individual symmetry classes, for which the above derived summations are made separately, on the ground that the

${ }^{4}$ J. O. Halford, J. Chem. Phys. 18, 444 (1950).
TABLE V. Constants in Eqs. 9-12.

\begin{tabular}{cccc}
\hline$T$ & $a$ & $b$ & $c$ \\
\hline 200 & -0.03741 & 0.14547 & -0.24410 \\
260 & -0.01929 & 0.05989 & -0.18779 \\
\hline
\end{tabular}

TABLE VI. $\theta$ values and symmetry classes.

\begin{tabular}{cccccc}
\hline & & & & & \\
$K$ & 0 & 1 & 2 & 3 & 4 \\
\hline$\pi \sigma / 3$ & $0^{\circ}(A)$ & $9.309\left(E_{1}\right)$ & $18.618\left(E_{2}\right)$ & $27.927(A)$ & $37.236\left(E_{1}\right)$ \\
$\pi(\sigma+1) / 3$ & $60^{\circ}\left(E_{1}\right)$ & $69.309\left(E_{2}\right)$ & $78.618(A)$ & $87.927\left(E_{1}\right)$ & $97.236\left(E_{2}\right)$ \\
$\pi(\sigma+2) / 3$ & $120^{\circ}\left(E_{2}\right)$ & $129.309(A)$ & $138.618\left(E_{1}\right)$ & $147.927\left(E_{2}\right)$ & $157.236(A)$ \\
\hline
\end{tabular}

TABLE VII. Internal rotator thermodynamic properties for methyl alcohol with $C_{1}=1.01 \times 10^{-40}, V_{0}=932 \mathrm{cal} . / \mathrm{mole}$. (cal. $/ \mathrm{deg}$. mole).

\begin{tabular}{ccccc}
\hline$T$ & $-F / T$ & $H / T$ & $S$ & $C$ \\
\hline 200 & 0.267 & 0.836 & 1.103 & 1.244 \\
260 & 0.499 & 0.931 & 1.430 & 1.241 \\
300 & 0.636 & 0.971 & 1.606 & 1.219 \\
340 & 0.759 & 0.999 & 1.758 & 1.197 \\
380 & 0.871 & 1.018 & 1.890 & 1.175 \\
420 & 0.974 & 1.032 & 2.006 & 1.155 \\
460 & 1.067 & 1.042 & 2.109 & 1.138 \\
500 & 1.156 & 1.049 & 2.205 & 1.123 \\
\hline
\end{tabular}

different symmetry classes may have different statistical weights arising from nuclear spin degeneracy. As might be predicted, this separation proves to be unnecessary in the temperature range under consideration. It can be done, however, with very little additional labor, and its inclusion adds weight to the validity of the results. The symmetry classification can be made by examining the quantum numbers of the limiting free rotator, since the presence of the barrier can change neither the symmetry class nor the order of occurrence of the levels. For the free rotator, the energy in arbitrary units is $\left(m-K C_{1} / C\right)^{2}$, where $m$ and $K$ are integers, and the symmetry class is determined by $K-m$. It is easily shown that, within each of the groups of states distinguished by a particular definition of $\theta$, each symmetry class will recur with each third value of $K$. If $S$ is an integer one symmetry class $(A)$ will have $K-m=3 S$, a second class $\left(E_{1}\right)$ will have $K-m=3 S+1$, and, for the third class $\left(E_{2}\right), K-m$ $=3 S+2$.

For those states for which $\theta=\pi \sigma / 3, m$ is always a multiple of 3 , and the symmetry is directly determined by $K$. At $K=0$, the symmetry is $A$, at $K=1$, it is $E_{1}$, and, throughout the series, starting with $K=0$, the order is $A E_{1} E_{2} A$ etc., within each of the bands of internal energy levels.

For the other two groups of levels, if $K=0, m= \pm 1$, for two states, $E_{1}$ and $E_{2}$, at the same energy, and the correspondence between symmetry and $\theta$ classification is not determined. When $\theta=\pi(\sigma+1) / 3$, the energy in the lowest band is greater for $K=1$ than for $K=0$, which is possible only if $m=-1$, and $K-m=2$, for symmetry $E_{2}$. If $K=2$, with still higher energy, $m=-1$ 
TABLE VIII. Extreme values of the thermodynamic properties of the internal rotator $\alpha^{2}=6.0553$ at $200^{\circ} \mathrm{K}$.

\begin{tabular}{ccccc}
\hline$\theta$ & $-F / T$ & $H / T$ & $S$ & $C$ \\
\hline 0 & 0.299 & 0.698 & 0.998 & 1.533 \\
$\pi / 2$ & 0.234 & 0.978 & 1.213 & 0.925 \\
\hline
\end{tabular}

TABLE IX. Internal rotator thermodynamic properties for methyl alcohol with $C_{1}=1.353 \times 10^{-40}, V_{0}=932 \mathrm{cal} . / \mathrm{mole}$. (cal./ deg. mole).

\begin{tabular}{ccccc}
\hline$T$ & $-F / T$ & $H / T$ & $S$ & $C$ \\
\hline 200 & 0.426 & 0.879 & 1.305 & 1.339 \\
260 & 0.671 & 0.981 & 1.652 & 1.302 \\
300 & 0.815 & 1.022 & 1.837 & 1.266 \\
340 & 0.944 & 1.049 & 1.993 & 1.233 \\
380 & 1.062 & 1.066 & 2.128 & 1.203 \\
420 & 1.169 & 1.078 & 2.247 & 1.177 \\
460 & 1.268 & 1.086 & 2.353 & 1.155 \\
500 & 1.358 & 1.090 & 2.449 & 1.137 \\
\hline
\end{tabular}

and $K-m=3$, for symmetry $A$. The symmetry order, starting with $K=0$, is therefore $E_{1} E_{2} A E_{1}$, etc. It is easily shown that for this definition of $\theta$ the same order holds for the higher internal energy bands.

By a like argument, or simply by inspection, the pattern of the states for which $\theta=\pi(\sigma+2) / 3$ is $E_{2} A E_{1} E_{2}$, etc., at all internal levels. Within each of these groups, the separation of states on the $\theta$ scale is $\pi C_{1} / 3 C$ and the separation of states of like symmetry is $\pi C_{1} / C$. It follows from the above considerations that a set of internal rotator levels derived for a particular value of $\theta$ will all fall in the same symmetry class. Consequently, within each symmetry class, each $K$ determines a value of $\theta$ which establishes a set of internal levels for which the sums $q, q^{\prime}$, and $q^{\prime \prime}$ can be evaluated as required by Eqs. (9).

For each value of $K$, there is then a value of $\theta$ for each symmetry class, and this value of $\theta$ leads to a complete set of internal rotator levels of the same symmetry, one state in each of the internal rotator bands. Within the limits of Eqs. (9), only the $\theta$ values are used as required by Eqs. (10)-(12). Otherwise, for greater accuracy, the summations $q_{K}, q_{K}{ }^{\prime}$, and $q_{K}{ }^{\prime \prime}$ could be evaluated individually and used as called for by Eqs. (4), (6), and (8).

Table VI illustrates the symmetry classification of the $\theta$ values and their association with $K$. The table is equally applicable to all the internal rotator bands. Within each of the three sets of levels, the interval between successive values of $\theta$ is $\pi C_{1} / 3 C=9.309$ degrees. The result, as shown below in Eqs. (13), is not sensitive to this interval, and would be obtained with a wide variety of intervals centered around the one used.

There is actually no need to distinguish between the $E_{1}$ and $E_{2}$ states, since there can never be any statistical difference between them. If a state at a given energy for $+K$ is an $E_{1}$ level, there will be an $E_{2}$ level at $-K$ with the same energy, with the result that the summations over all values of $K$ will be the same for both symmetry classes. This follows from the energy of the free rotator, $\left(m-K C_{1} / C\right)^{2}$. If the signs of both $m$ and $K$ are reversed, the energy is invariant. If $K-m$ is a multiple of 3 , then $m-K$ is also a multiple of 3 and the symmetry class $(A)$ is unchanged. If, however, $K-m$ is $3 S_{1}+1, m-K$ will be $3 S_{2}+2$, for a change of symmetry from $E_{1}$ to $E_{2}$ by the reversal of signs. The situation is not changed by the presence of the barrier, with the result that the pattern of $E_{1}$ states for $+K$ is the same as that of the $E_{2}$ states for $-K$, with the two coinciding at $K=0$.

From Table VI, in an extended form, the $\theta$ values required by Eqs. (10)-(12) can be selected. When the indicated sums are evaluated at 200 and $260^{\circ} \mathrm{K}$, it is found, independently for each symmetry class, that

$$
\begin{gathered}
\sum_{K}\left(\sin ^{2} \theta_{K}\right) \exp \left(-K^{2} \beta\right) / Q_{K}=0.50000 \\
\sum_{K}\left(\sin ^{2} \theta_{K}\right) K^{2} \beta \exp \left(-K^{2} \beta\right) / Q_{K}=0.25000 \\
\sum_{K}\left(\sin ^{2} \theta_{K}\right) K^{4} \beta^{2} \exp \left(-K^{2} \beta\right) / Q=0.3750 .
\end{gathered}
$$

Consequently the thermodynamic properties do not vary with the symmetry class and are therefore calculable without knowledge of the actual spin degeneracies. The result obtained for any one symmetry class can be used to represent the whole system, or the summations could have been made over the whole set of states and subsequently adjusted by means of the symmetry number.

When the sums of Eqs. (13) are introduced into Eqs. (10)-(12), the results are

$$
\bar{q}=q_{0}+a / 2 ; \bar{q}^{\prime}=q_{0}{ }^{\prime}+b / 2 \text { and } \bar{q}^{\prime \prime}=q_{0}{ }^{\prime \prime}+c / 2 \text {, }
$$

that is, the valid average sum is in each case the numerical mean of the sums for $\theta=0$ and $\theta=\pi / 2$. It is evident that the thermodynamic properties will not be far from the numerical mean of the extreme calculable values.

It is of interest to inquire how general the result of Eqs. (13) and (14) may be. Further consideration reveals that, although there are a variety of geometrical situations for which the results would be at least fairly accurate, there are other situations for which the correct average results might be considerably different. If one of two coaxial rotators remains fixed while the other one is increased in size, it develops that, although the number of populated $K$ states increases, the coverage of the cycle in $\theta$ or $\sigma$ becomes less. Since the simple numerical results of Eqs. (13) and (14) can be obtained only with sufficient coverage of this cycle, it follows that a situation will eventually be reached in which the valid $\bar{q}$ for each symmetry class approaches the value of $q$ for $K=0$. In a molecule like tert. butyl alcohol, where presumably only $A$ states of the hydroxyl rotation could exist, the thermodynamic properties would approach those calculable for $\theta=0$, namely, those derived from $q_{0}, q_{0}{ }^{\prime}$, and $q_{0}{ }^{\prime \prime}$. In the extreme case of a small group rotating against an infinitely large one, this limit would actually be reached. It will be necessary to 
TABLE X. Comparison with experimental heat capacities.

\begin{tabular}{cccccc}
\hline \hline$T$ & $C_{t+r}$ & $C_{\text {vib }}$ & $C_{r r}$ & $C_{v}{ }^{\circ}$ (calc.) & $C_{v^{\circ}(E \text { and } F)}$ \\
\hline 200 & 5.962 & 0.141 & $1.244,1.339$ & $7.347,7.442$ & $7.45 \pm 0.11$ \\
280 & 5.962 & 0.855 & $1.230,1.284$ & $8.047,8.101$ & $8.38 \pm 0.08$ \\
& & & & & $C_{v}{ }^{\circ}$ (Rowlinson) \\
338 & & & & 8.95 & 8.9 \\
351 & & & & 9.18 & 9.2 \\
362 & & & & 9.37 & 9.7 \\
383 & & & & 9.75 & 10.0 \\
406 & & & & 10.19 & 10.3 \\
448 & & & 11.01 & 11.7 \\
\hline \hline
\end{tabular}

take an average only when $C_{1}$ is small or $T$ is very low, and the numerical mean sums validated here for methyl alcohol will be accurate only if $C$ is also small so that $C_{1} / C$ is appreciable.

The mean thermodynamic properties of the internal rotation are calculated from Tables II to $\mathrm{V}$, inclusive, as shown in Table VII. At 200 and $260^{\circ}$ the complete summations leading to the results of Eqs. (13) have been carried through, but at the higher temperatures only the extreme values of the sums have been used, in accordance with Eqs. (14).

As an example of the spread of the values for which the average has been found, the extreme thermodynamic properties at $200^{\circ}$ are shown in Table VIII. Each range decreases with the temperature until at $500^{\circ}$ the spread in each property has almost completely disappeared. At $200^{\circ}$ the correct average heat capacity differs from the numerical mean of the extremes by 0.015 , but is almost exactly equal to the result derived from a set of energy levels calculated for $\theta=\pi / 4$, that is, to the result obtained from the mean $\theta$ for a half-cycle.

Because there is still some doubt about the correct value for $C_{1}$, the moment of inertia of the $\mathrm{OH}$ group, or, in other words, about the size of the $\mathrm{COH}$ angle, another set of properties has been calculated for $\alpha^{2}=10.0000$, which corresponds to a reduced internal moment of 1.0859 , and to $C_{1}=1.353\left(\times 10^{-40}\right)$. For this case the $\mathrm{COH}$ angle would be slightly larger than the accepted internal angle of the water molecule. The required energy levels at $\theta=0$ and $\theta=\pi / 2$, have been tabulated elsewhere. ${ }^{4}$ The corresponding contributions to the thermodynamic properties appear in Table IX.

In the upper part of Table $\mathrm{X}$ heat capacities from molecular constants are compared with the low pressure experimental results of Eucken and Franck, ${ }^{5}$ obtained from the thermal conductivity of the vapor at 200 and $280^{\circ} \mathrm{K}$. Under $C_{r r}$ and $C_{V}{ }^{\circ}$, two values are given which correspond, respectively, to the lower and higher moments of inertia which provide the bases for Tables VII and IX. In the lower part of Table X, the heat capacities of Rowlinson, ${ }^{6}$ obtained from the ve-

\footnotetext{
S A. Eucken and E. U. Franck, Zeits. f. Elektrochemie 52, 195 (1948).

G J. S. Rowlinson, Nature 162, 820 (1948).
}

TABLE XI. Entropy of methyl alcohol, hypothetical perfect gas, with $C_{1}=1.01 \times 10^{-40}, V_{0}=932 \mathrm{cal} . / \mathrm{mole}$. (cal. $/ \mathrm{deg}$. mole).

\begin{tabular}{cccccc}
\hline$T$ & $S(t+r)$ & $S$ (vib) & $S_{r r}$ & $S^{\circ}$ (1 atmos.) & $S$ (third law) \\
\hline 200 & $\mathbf{5 2 . 1 4 2}$ & 0.019 & 1.103 & $\mathbf{5 3 . 2 6 4}$ & $\mathbf{5 1 . 2 8 6}$ \\
260 & $\mathbf{5 4 . 2 8 2}$ & 0.103 & 1.430 & 55.815 & $\mathbf{5 5 . 5 1 1}$ \\
300 & $\mathbf{5 5 . 3 6 5}$ & 0.223 & 1.606 & $\mathbf{5 7 . 1 9 4}$ & $\mathbf{5 6 . 9 4 4}$ \\
340 & $\mathbf{5 6 . 3 6 0}$ & 0.405 & 1.758 & 58.523 & $\mathbf{5 8 . 2 7 3}$ \\
380 & 57.244 & 0.644 & 1.890 & 59.778 & 59.489 \\
420 & $\mathbf{5 8 . 0 4 0}$ & 0.937 & 2.006 & 60.983 & 60.578 \\
460 & $\mathbf{5 8 . 7 6 3}$ & 1.274 & 2.109 & 62.146 & 61.381 \\
$\mathbf{5 0 0}$ & $\mathbf{5 9 . 4 2 9}$ & $\mathbf{1 . 6 5 2}$ & 2.205 & 63.282 & 61.079 \\
\hline
\end{tabular}

locity of supersonic waves, as read approximately from his plotted points, are compared with statistical heat capacities taken by linear interpolation from totals derived from the mean values of Tables VII and IX.

The agreement between calculation and experiment is good enough to suggest that the molecular constants are nearly correct, but does not fully substantiate them and certainly provides no good basis for a choice between the alternative values of $C_{1}$. In addition, an attempt was made to reduce the calorimetric heat capacities of DeVries ${ }^{7}$ and Collins to the limiting perfect gas values. The results obtained by using the equation of state proposed by Eucken and Meyer ${ }^{8}$ were in fair agreement with calculated values only at the higher temperatures, but the over-all comparison with calculation was not instructive enough to justify separate tabulation.

In Table XI, the third law entropy, calculated as previously described, ${ }^{1}$ is compared with the entropy from molecular constants obtained with $C_{1}=1.01$ $\times 10^{-40}$. At the extreme high and low temperatures there are evidently errors in the third law values due to extrapolation of the vapor pressures and heats of vaporization, and to the use of the equation of state beyond its range of validity. Between $260^{\circ}$ and $380^{\circ} \mathrm{K}$, however, there is a nearly constant difference between the molecular and third law values, which lies at the extreme limit of estimated errors. The substitution of $C_{1}=1.35 \times 10^{-40}$ adds approximately 0.30 entropy unit to this difference, and suggests that there is a residual entropy or at least a fairly large constant error. As compared with the earlier estimate of barrier height, the elimination of the extrapolation of the tables of Pitzer and Gwinn has increased the range of consistency by about $50^{\circ}$.

From the above calculations, it appears that the required molecular constants are known well enough to determine the limiting low pressure heat capacity quite closely, and to fix the hypothetical perfect gas entropy at any one temperature within about 0.30 cal./deg. mole. Vapor densities, however, are not well enough established to give reliable properties of the

${ }^{7}$ T. DeVries and B. T. Collins, J. Am. Chem. Soc. 63, 1343 (1941).

${ }^{8}$ A. Eucken and L. Meyer, Zeits. f. physik. Chemie 5B, 452 (1929). 
real vapor over any considerable range of pressures. The third law entropy appears to contain a constant discrepancy between 0.25 and $0.55 \mathrm{cal} . / \mathrm{deg}$. mole, part of which, however, might be eliminated by more accurate vapor density data. Provisionally, the molecular constants used to obtain Tables I-VIII, X, and XI should give accurate enough limiting perfect gas thermodynamic properties for most purposes.

\title{
On the Vibrational Spectra of Crystals
}

\author{
Wayne A. Bowers and Herbert B. Rosenstock \\ University of North Carolina, Chapel Hill, North Carolina
}

(Received March 27, 1950)

\begin{abstract}
In order to check the accuracy of distributions of characteristic vibration frequencies in crystals as calculated by approximate methods proposed by various workers, the frequency distribution is calculated rigorously for a lattice for which this is possible, viz., a two-dimensional square lattice with vibrations perpendicular to the lattice plane. The calculation is then repeated by the use of the various approximate methods, and comparisons are drawn. The effect of using any of the approximate distributions in place of the exact one in the calculation of thermodynamic functions is illustrated by a calculation of the specific heat of this same crystal. Finally, an extension to three dimensions is discussed.
\end{abstract}

\section{INTRODUCTION}

$\mathbf{I}^{\mathrm{N}}$ $\mathrm{N}$ order to calculate the specific heat and other thermodynamic functions of a crystalline solid, it is necessary to know the distribution of natural frequencies of the lattice vibrations. Although the fundamental equations for the natural frequencies were written down by Born and von Karman in 1912, the mathematical complexities have prevented the actual calculation of frequency distributions except by laborious numerical procedures for particular values of the parameters involved. For this reason one has to rely on various approximations to the actual frequency distribution, of which the most famous one is, of course, the Debye theory, which approximates the crystal by a continuous medium and uses the distribution of elastic vibrations in a continuum, with a cut-off adjusted to give the correct total number of vibrations. The remarkable success of the Debye theory in explaining observed specific heat data put the question of rigorous calculation of frequency distributions in the background for a number of years, until Blackman, ${ }^{2}$ in a series of papers, showed that certain anomalies in observed specific heat data could be at least qualitatively explained in terms of the deviation of the actual frequency distribution from the Debye distribution. Since that time a number of workers $^{3-5}$ have devoted themselves to working out approximate methods of calculating frequency distributions from the Born-von Karman equations.

The purpose of this paper is to compare the distributions obtained by various approximate methods with the actual one in a case for which an expression for it can

\footnotetext{
${ }^{1}$ M. Born and T. von Karman, Physik. Zeits. 13, 297 (1912).

2 M. Blackman, Proc. Roy. Soc. 148, 365 (1935); 159, 416 (1937).

${ }^{3}$ E. W. Montroll, J. Chem. Phys. 10, 218 (1942); 11, 481 (1943)

4 W. V. Houston, Rev. Mod. Phys. 20, 161 (1948).

${ }^{5}$ P. C. Fine, Phys. Rev. 56, 355 (1939).
}

be obtained in closed form, namely, the case of vibrations of a square two-dimensional lattice in a direction transverse to its own plane. Although this is a case of no direct physical significance (cf. Section $V$ ), the existence of an analytic solution makes possible a comparison with approximate methods and hence gives one some basis for evaluating these methods. Further, certain conclusions with regard to actual three-dimensional lattices may be drawn.

In Section II we formulate the two-dimensional problem mentioned above and derive the actual frequency distribution; in Section III we compare the actual distribution with the distributions of Debye, Montroll, ${ }^{3}$ and Houston ; ${ }^{4}$ in Section IV we calculate the specific heat of the crystal, using both the exact and the approximate frequency distributions; and in Section V we discuss a three-dimensional problem for which a rigorous result can be obtained.

\section{TRANSVERSE VIBRATIONS OF A SQUARE LATTICE}

Consider a square lattice consisting of $N$ rows and $N$ columns of identical atoms. If the displacement, transverse to the plane of the lattice, of the atom in the $l$ th row and $m$ th column is denoted by $u_{l, m}$, we may write the Lagrangian function:

$$
\begin{aligned}
L= & \frac{1}{2} M \sum_{l, m=1}^{N} \dot{u}_{l, m}{ }^{2} \\
& -\frac{1}{2} \alpha\left\{\sum_{l, m=1}^{N}\left(u_{l, m+1}-u_{l, m}\right)^{2}+\sum_{l, m=1}^{N}\left(u_{l+1, m}-u_{l, m}\right)^{2}\right\} \\
& -\frac{1}{2} \gamma\left\{\sum_{l, m=1}^{N}\left(u_{l+1, m+1}-u_{l, m}\right)^{2}\right. \\
& \left.+\sum_{l, m-1}^{N}\left(u_{l+1, m-1}-u_{l, m}\right)^{2}\right\} .
\end{aligned}
$$

